\title{
Effects of Preexisting Psychotropic Medication Use on a Cohort of Patients with Ischemic Stroke Outcome
}

\author{
Adalia H. Jun-O'Connell ${ }^{1},{ }^{1}$ Dilip K. Jayaraman, ${ }^{2}$ Nils Henninger, ${ }^{1,3}$ Brian Silver, ${ }^{1}$ \\ Majaz Moonis, ${ }^{1}$ and Anthony J. Rothschild ${ }^{3}$ \\ ${ }^{1}$ Departments of Neurology, University of Massachusetts Medical School, Worcester, MA, USA \\ ${ }^{2}$ Neurology Department, Tower Health Medical Group, University of Massachusetts Medical School, Worcester, MA, USA \\ ${ }^{3}$ Department of Psychiatry, University of Massachusetts Medical School, Worcester, MA, USA
}

Correspondence should be addressed to Adalia H. Jun-O’Connell; adalia.jun@umassmemorial.org

Received 27 April 2020; Revised 28 July 2020; Accepted 16 August 2020; Published 22 September 2020

Academic Editor: Augusto Fusco

Copyright (c) 2020 Adalia H. Jun-O’Connell et al. This is an open access article distributed under the Creative Commons Attribution License, which permits unrestricted use, distribution, and reproduction in any medium, provided the original work is properly cited.

Background. Several studies investigated the use of selective serotonin reuptake inhibitors (SSRI) after ischemic stroke to improve motor recovery. However, little is known about the effects of preexisting psychotropic medication use (PPMU), such as antidepressants, on a long-term ischemic stroke functional disability. Objective. We sought to determine the prevalence of PPMU and whether PPMU relates to the long-term clinical outcome in a cohort of patients presenting with acute ischemic strokes. Methods. We retrospectively analyzed 323 consecutive patients who presented with an acute ischemic stroke in a single institution between January 2015 and December 2017. Baseline characteristics, functional disability as measured by the modified Rankin Scale (mRS), and major adverse cardiovascular complications (MACE) within 365 days were recorded. The comparison groups included a control group of ischemic stroke patients who were not on psychotropic medications before and after the index ischemic stroke and a second group of poststroke psychotropic medication use (PoMU), which consisted of patients started on psychotropic medication during the index admission. Results. The prevalence of PPMU in the studied cohort was $21.4 \%$ (69/323). There was a greater proportion of females in the PPMU than in the comparison groups $(P<0.001)$, while vascular risk factors were similar in all groups, except for an increased presence of posterior circulation infarcts in the PPMU $(37.4 \%$ vs. $18.8 \%, P<0.001)$. Among the patients with available 1 -year follow-up data $(n=246)$, we noted significantly greater improvement in stroke deficits, measured by National Institute of Health Stroke Scale (NIHSS) between PPMU and PoMU vs. control (3 (0-7) versus $1(0-4), P=0.041)$. The 1-year mRS was worse in PPMU and PoMU compared to the control group (2 (IQ 1-3) vs. 2 (IQ 0-3) vs. 1 (IQ 0-2), respectively, $P=0.013$ ), but delta mRS reflecting the degree of mRS improvement showed no significant difference between any PMU and control patients $(P=0.76)$. There was no statistically significant difference in MACE. Conclusion. PPMU in ischemic stroke is common; it can be beneficial in ischemic stroke in the long-term clinical outcome and is not associated with increased risks of MACE.

\section{Introduction}

Stroke is the third leading cause of worldwide disability [1] and a leading cause of serious long-term disability in the US, reducing mobility in more than half of its survivors aged 65 and older [2]. Motor deficits affect up to $82 \%$ of patients after stroke and are associated with decreased quality of life
[3]. Several neurotransmitters, specifically norepinephrine [4] [5] [6], dopamine [7] [8], and serotonin [9] [10, 11] have been implicated in the modulation of motor recovery after brain injury. A meta-analysis of combined animal and human researches suggests that antidepressants, such as selective serotonin reuptake inhibitors (SSRI), play a crucial role in poststroke neurological recovery through its probable 
action on a regenerative process [12]. Subsequently, there has been an increased interest in the role of psychotropic drugs to potentially improve poststroke motor recovery [3].

Preexisting psychotropic medication use (PPMU) prior to the onset of an ischemic stroke on long-term stroke outcome is unknown. SSRI have been studied in several randomized control studies, yielding conflicting results regarding the effect of the medication on outcomes after an ischemic stroke $[11,13-15]$. Furthermore, the effects of PPMU on acute stroke severity and poststroke outcome have not been well studied. To our knowledge, there has been only one cohort study that investigated the association between SSRI use prior to an ischemic stroke and subsequent outcome within 30 days [16].

In the current study, we sought to determine the prevalence of PPMU in a cohort of patients presenting with an acute ischemic stroke and whether PPMU was associated with the initial stroke severity as assessed by the admission National Institute of Health Stroke Scale (NIHSS) score. Secondary objectives were to determine the association between PPMU with long-term functional disability as assessed on the modified Rankin Scale (mRS), as well as major adverse cardiovascular events (MACE).

\section{Methods}

2.1. Study Cohort. The study was approved by the local Institutional Review Board, and a Health Insurance Portability and Accountability Act waiver of informed consent was approved. We retrospectively analyzed prospectively accrued adult patients (greater than age 18 years) who were evaluated at the University of Massachusetts Medical Center between January 2015 and December 2017. We followed the Strengthening the Reporting of Observational Studies in Epidemiology guidelines (http://www.strobe-statement.org) [17].

2.2. Definitions. We defined ischemic stroke as an episode of neurological dysfunction due to a focal CNS infarction, attributable to ischemia [18].

Psychotropic medications were defined as drugs used to treat psychiatric disorders [19]. The specific psychotropic medications examined in this study included drugs that target dopaminergic, norepinephrinergic, and serotonergic neurotransmitters, including antidepressants (tricyclic antidepressants, SSRIs, serotonin-norepinephrine reuptake inhibitors, serotonin antagonist and reuptake inhibitors, monoamine oxidase inhibitors), amphetamines, lithium, and select atypical antipsychotics (quetiapine, aripiprazole, and ziprasidone for examples, which may enhance serotonergic transmission) [19].

Based on the use of psychotropic medication before and after the index stroke, we stratified patients to three groups (Figure 1): first, preexisting psychotropic medication use (PPMU), which included patients who were treated with a psychotropic medication prior to the onset of the acute ischemic stroke and were continued on them after the index stroke; second, poststroke psychotropic medication use (PoMU), which included patients who were started on psychotropic medication during the index admission; and third, control, which included patients who were neither treated with psychotropic medications before nor after the index stroke. To minimize potential expectation bias, a subclassification of study groups was carried out blind to any follow-up data.

A favorable 1-year functional outcome was defined as a modified Rankin Scale (mRS) score $\leq 2$ [20]. We defined the degree of functional deficit recovery as the difference between the admission NIHSS minus the 1-year NIHSS score (delta NIHSS), whereby larger numbers indicate greater deficit improvement. The $\mathrm{mRS}$ assessment in the poststroke period was assessed by a stroke-trained physician certified in $\mathrm{mRS}$ via in-person. When the mRS was not available, the $\mathrm{mRS}$ was reconstructed from the case description based upon the $\mathrm{mRS}$ criteria [21]. All diagnoses were first established by treating physicians and then reassessed by a trained vascular neurologist (D.J.) after independent chart reviews of the medical records. A double-boarded psychiatrist and neurologist (A.J.O.) adjudicated uncertain cases, and remaining discrepancies were resolved by consensus.

2.3. Exclusion. We excluded patients with severe stroke deficits (NIHSS $>20$ ) with expected poor prognosis, substantial premorbid disability $(\mathrm{mRS}>4)$, patients who died in house or were discharged to hospice. Patients lost to follow-up were excluded from our exploratory analyses.

2.4. Data Collection. Patient demographics, comorbidities, preadmission medications, admission NIHSS, admission mRS, and imaging data (MRI brain and/or CT head) were collected for all patients by neurology trained physicians [22]. All included patients $(n=323)$ underwent a brain CT. An additional imaging with brain MRI $(n=301,93.2 \%)$ was done at the discretion of the treating physician at the time of the index admission.

2.5. Outcomes. The primary goals were to identify the prevalence of PPMU in acute ischemic stroke and its association with admission stroke severity as graded by NIHSS. For the purpose of this analysis, we compared PPMU with nonPPMU (controls + PoMU) patients.

In a subsequent exploratory analysis, we sought to determine whether psychotropic medication use was associated with 1-year disability (defined as $\mathrm{mRS}>2$ ), delta $\mathrm{mRS}$ (degree of mRS improvement from baseline to 1 year to account for the degree of prestroke mRS), delta NIHSS (degree of NIHSS score improvement from baseline to 1 year to account for potential confounding by indication as patients with worse acute NIHSS were more likely started on psychotropic medication), and major adverse cardiac events (MACE) and its individual components of recurrent nonfatal stroke, nonfatal myocardial infarction, and cardiovascular death. Given prior clinical trial data suggesting beneficial effect on motor recovery with PoMU [11], we conducted an additional exploratory analysis to determine whether any psychotropic medication use (any PMU, i.e., combined PPMU and PoMU) was associated with improved 1-year disability and neurological deficit recovery (as assessed by the delta NIHSS) at one year when compared to controls. 


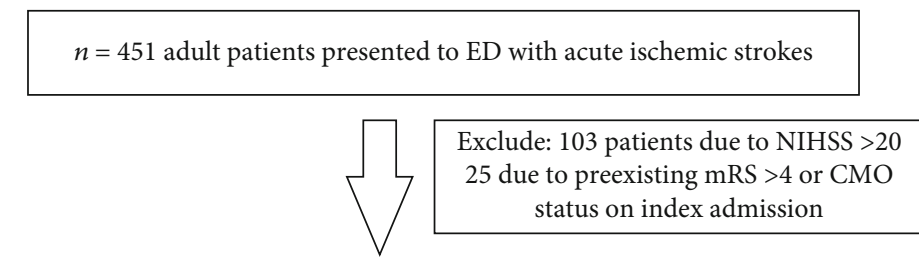

Primary analysis $(n=323)$ : prevalence of preadmission psychotropic medication use and its association with admission stroke severity (control [ $n=226]$, PPMU [ $n=69]$, PoMU [ $n=28]$ ).

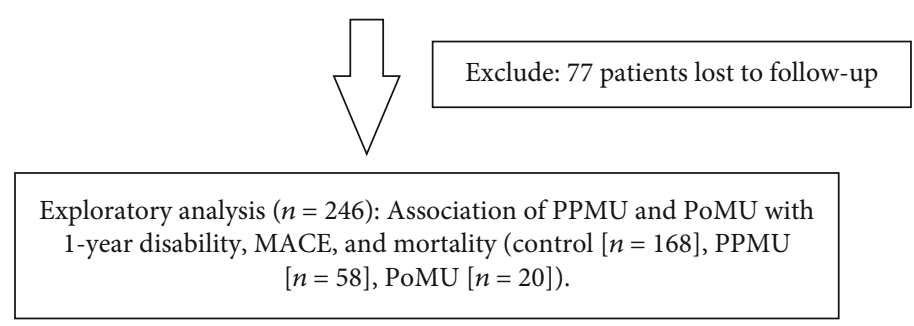

Figure 1: Patient flow chart.

2.6. Statistical Analyses. Data are reported as median (interquartile range) unless otherwise stated. Univariate comparisons were performed with $\chi^{2}$, Fisher exact, MannWhitney $U$ tests, and Kruskal Wallis ANOVA on ranks as appropriate. Two-sided significance tests were used throughout, and a two-sided $P<0.05$ was considered statistically significant unless stated otherwise. To calculate corrected levels of significance in cases of multiple comparisons in the univariate analyses, adjusted significance level was calculated using the Bonferroni correction. All statistical analyses were performed using the IBM SPSS Statistics version 20.0.0 (IBM, Armonk, NY).

\section{Results}

3.1. Study Participants and Prevalence of Psychotropic Medication Use. Overall, 323 patients fulfilled the study criteria and were included in the data analysis (Figure 1). Of these, 69 (21.4\%) patients were on PPMU, $28(8.7 \%)$ patients were on PoMU, and 226 (69.9\%) patients served controls. The details on the used psychotropic medications stratified by PPMU and PoMU are shown in Table 1.

3.2. Clinical Characteristics Associated with PPMU. The baseline characteristics of the studied patient population as stratified by PPMU versus non-PPMU are shown in Table 2. The prevalence of female gender in PPMU was higher than in other groups $(68.1 \%$ vs. $37.4 \%, P<0.001)$. Moreover, the PPMU group had similar prevalence of preexisting vascular risk factors compared to the non-PPMU group, including hypertension, diabetes mellitus, prior history of TIA and stroke, atrial fibrillation, coronary artery disease, and peripheral vascular disease $(P>0.05)$, and similar use of stroke prevention medications $(P>0.05)$.

3.3. Association of PPMU with Initial Stroke Severity. Overall, baseline characteristics of PPMU and non-PPMU groups were similar except for a higher preadmission mRS and more frequent posterior circulation stroke location in PPMU patients $(P<0.05$, each, Table 2$)$. With regard to the initial stroke severity, we found no significant difference in the admission NIHSS between PPMU and non-PPMU groups (median 3 (IQ 1-8.5) vs. 3 (IQ 1-7), $P=0.352$ ). Similarly, there was no difference in the final infarct volume and length of hospital stay $(P>0.05$, each).

3.4. Exploratory Analysis of 1-Year Outcome Events. A total of $246(76.2 \%)$ patients had 1-year outcome data available for analysis. There was no significant difference in the proportion of patients lost to follow-up between groups $(P>0.05)$. Table 3 depicts the secondary outcome events in the three defined groups.

We found that the clinical deficit severity as measured by the NIHSS at one year was significantly worse in PoMU compared to control and PPMU patients, respectively (median 1.5 vs. 0 and $1 ; P=0.035)$. To account for potential confounding by indication (i.e., patients with worse acute NIHSS were more likely started on psychotropic medication), we also examined the degree of NIHSS improvement from admission to 1 year (delta NIHSS). In this analysis, there was a trend towards greater NIHSS improvement between PPMU and PoMU versus control, though this did not reach significance (Table 3). In a separate analysis of any PMU versus control, any PMU patients had an overall greater focal deficit recovery than controls as assessed by the delta NIHSS (3 (0-7) versus $1(0-4), P=0.041)$.

When we examined the degree of functional disability as measured by the mRS, we found that patients in both the PPMU and PoMU had significantly worse 1 -year mRS than the control group $2(1-3)$ vs. $2(0-3)$ vs. $1(0-2)$, respectively, $P=0.013)$. When we accounted for the degree of prestroke $\mathrm{mRS}$ by calculating the degree of $\mathrm{mRS}$ improvement from baseline to 1 year (delta mRS), this effect was attenuated when compared across all three groups $(P=0.046)$, and there 
TABLE 1: Frequency of psychotropic medication use.

(a)

\begin{tabular}{lc}
\hline Preischemic stroke psychotropic medication name & $n=69$ \\
Monotherapy & 56 \\
Sertraline & 6 \\
Amitriptyline & 3 \\
Venlafaxine & 7 \\
Duloxetine & 0 \\
Citalopram & 11 \\
Trazodone & 9 \\
Olanzapine & 1 \\
Nortriptyline & 1 \\
Fluoxetine & 8 \\
Pramipexole & 1 \\
Mirtazapine & 2 \\
Sinemet & 1 \\
Bupropion & 1 \\
Quetiapine & 1 \\
Paroxetine & 1 \\
\hline Polytherapy & 4 \\
Postischemic stroke psychotropic medication use & 13 \\
Monotherapy & $n=28$ \\
Escitalopram & 25 \\
Quetiapine & 1 \\
Amitriptyline & 1 \\
Depakote & 1 \\
Citalopram & 1 \\
Trazodone & 2 \\
Paroxetine & 2 \\
Fluoxetine & 1 \\
\hline Polytherapy & 1 \\
\hline
\end{tabular}

(b)

\begin{tabular}{lc}
\hline $\begin{array}{l}\text { Polytherapy } \\
\text { Preischemic stroke } \\
\text { case \# }(n=13)\end{array}$ & Medication names \\
\hline 1 & Sertraline, methylphenidate \\
2 & Duloxetine, quetiapine \\
3 & Olanzapine, lithium \\
4 & Citalopram, risperidone \\
5 & Fluoxetine, trazodone \\
6 & Divalproex sodium, trazodone, bupropion, \\
7 & methylphenidate \\
8 & Amitriptyline, fluoxetine \\
9 & Buspirone, sertraline \\
10 & Quetiapine, mirtazapine \\
\hline
\end{tabular}

TABLE 1: Continued.

\begin{tabular}{lc}
\hline $\begin{array}{l}\text { Polytherapy } \\
\text { Preischemic stroke } \\
\text { case \# }(n=13)\end{array}$ & Medication names \\
\hline 11 & Amitriptyline, mirtazapine, trazodone \\
12 & Sertraline, trazodone \\
13 & Escitalopram, trazodone \\
Postischemic stroke & Medication names \\
case \# $(n=3)$ & Citalopram, trazodone \\
1 & Fluoxetine, trazodone \\
2 & Olanzapine, trazodone \\
3
\end{tabular}

was no significant difference between any PMU and control patients $(P=0.76)$.

Finally, we found no statistically significant difference between the 3 groups in the rates of 12-month MACE (recurrent nonfatal stroke, nonfatal myocardial infarction, and cardiovascular death) $(P>0.05$, each, Table 3$)$. The results were not meaningfully different when we compared controls with any PMU (not shown).

\section{Discussion}

Our study is important as it addresses the potential association between preadmission psychotropic medication use in ischemic stroke and long-term disability in a select cohort of patients presenting with ischemic strokes. This is relevant as psychotropic medication in stroke is becoming more recognized due to the increasing awareness of psychiatric complications in strokes and the utilization of psychotropic medications towards recovery $[11-15,23]$. In our study, we found an association between greater functional deficit recovery and any psychotropic medication use (pre- or poststroke) in ischemic stroke compared to control, which leads to an important suggestion that psychotropic medication use may be beneficial in ischemic stroke. Our data also suggests that overall median mRS was still favorable (mRS score $\leq 2$ ) for each group and that psychotropic medication use does not appear to increase the risks of MACE.

There are important implications related to prepsychotropic medication use in ischemic stroke. Preexisting SSRI use and poststroke recovery have not been widely studied. Thus far, there have been two studies, with one study looking at discharge mRS from the index admission [20] and one looking at the risk of stroke mortality and morbidity within 30 days [16]. In these studies, pre-SSRI use in ischemic stroke patients was associated with good clinical outcomes at early follow-up following acute ischemic stroke and that prestroke SSRI use was not associated with an increased risk of severe stroke or mortality within 1 month, respectively. Moreover, although the initiation of SSRI in an acute ischemic stroke and its effect on poststroke recovery has been studied in several randomized control studies, the results are conflicting $[11,13-15]$ as only one study with a smaller sample size was 
TABle 2: Patient characteristics.

\begin{tabular}{|c|c|c|c|}
\hline Characteristics & Prepsychotropic use $(n=69)$ & Non-PPMU (control +PoMU) $(n=254)$ & Unadjusted $P$ value \\
\hline Gender, female & $47(68.1 \%)$ & $95(37.4 \%)$ & $<0.001$ \\
\hline Age & $66(57-76)$ & $67(58-78)$ & 0.443 \\
\hline Admission NIHSS & $3(1-8.5)$ & $3(1-7)$ & 0.352 \\
\hline MRS on index presentation & $0(0-2)$ & $0(0-0)$ & $<0.001$ \\
\hline IV tPA on index presentation & $12(17.4 \%)$ & $62(24.4 \%)$ & 0.26 \\
\hline Thrombectomy & $9(13.0 \%)$ & $25(9.8 \%)$ & 0.442 \\
\hline Decompressive craniectomy & $1(1.4 \%)$ & $7(2.8 \%)$ & 0.536 \\
\hline Length of stay & $4(3-7.5)$ & $4(3-7)$ & 0.731 \\
\hline Stroke volume $(\mathrm{ml})$ & $1.4(1.0-7.0)$ & $1.6(1.0-13)$ & 0.312 \\
\hline Discharge destination & & & 0.343 \\
\hline Home & $32(46.4 \%)$ & $115(45.3 \%)$ & \\
\hline Acute rehab & $26(37.7 \%)$ & $113(44.5 \%)$ & \\
\hline Skilled nursing facility & $11(15.9 \%)$ & $26(10.2 \%)$ & \\
\hline \multicolumn{4}{|l|}{ Preexisting risk factors } \\
\hline HTN & $51(73.9 \%)$ & $177(69.7 \%)$ & 0.494 \\
\hline Dyslipidemia & $47(68.1 \%)$ & $155(61.0 \%)$ & 0.280 \\
\hline $\mathrm{DM}$ & $23(33.3 \%)$ & $75(29.5 \%)$ & 0.542 \\
\hline History of TIA/stroke & $23(33.3 \%)$ & $66(26.0 \%)$ & 0.226 \\
\hline Atrial fibrillation & $8(11.6 \%)$ & $49(19.3 \%)$ & 0.137 \\
\hline CAD & $16(23.2 \%)$ & $54(21.3 \%)$ & 0.730 \\
\hline PVD & $14(20.3 \%)$ & $46(18.1 \%)$ & 0.680 \\
\hline \multicolumn{4}{|l|}{ Preadmission medications } \\
\hline Statins & $42(60.9 \%)$ & $121(47.6 \%)$ & 0.051 \\
\hline Antihypertensives & $47(68.1 \%)$ & $156(61.4 \%)$ & 0.307 \\
\hline Antidiabetics & $17(24.6 \%)$ & $58(22.8 \%)$ & 0.753 \\
\hline Antiplatelet & $31(44.9 \%)$ & $122(48.0 \%)$ & 0.647 \\
\hline Anticoagulation & $5(7.2 \%)$ & $18(7.1 \%)$ & 0.964 \\
\hline Lesion side & & & 0.756 \\
\hline Right & $31(44.9 \%)$ & $12(44.1 \%)$ & \\
\hline Left & $30(43.5 \%)$ & $103(40.6 \%)$ & \\
\hline Both & $7(10.1 \%)$ & $29(11.4 \%)$ & \\
\hline Lesion circulation & & & 0.001 \\
\hline Anterior & $46(66.7 \%)$ & $143(56.3 \%)$ & \\
\hline Posterior & $13(18.8 \%)$ & $95(37.4 \%)$ & \\
\hline Both & $9(13.0 \%)$ & $7(2.8 \%)$ & \\
\hline Hemorrhagic conversion & $6(8.7 \%)$ & $22(8.7 \%)$ & 0.993 \\
\hline
\end{tabular}

Data are median (IQ range) and $n$ (\%).

able to demonstrate a positive effect [11]. Future studies may benefit from evaluating prestroke measures, including prestroke psychotropic medication use as it may potentially complement other treatment strategies to improve stroke recovery [20]. Another important implication is the role of female gender in ischemic stroke and prepsychotropic medication use. In a pooled analysis of 19,652 patients, women were observed to have a higher disability and lesser quality of life following an ischemic stroke compared to men [24]. Furthermore, it is known that women have a higher prevalence of poststroke depression compared to men [25]. Poststroke depression is a serious and yet common complica- tion of stroke, with more severe symptoms of poststroke depression occurring in women, which can be associated with higher mortality rates [23].

Poststroke recovery is known to involve activation of the mechanisms for plasticity in adjacent neurons through longterm potentiation (LTM) [26, 27]. Several neurotransmitters are known to be involved in LTM and the modulation of motor recovery after brain injury, including norepinephrine $[6,28,29]$, dopamine $[7,8]$, and serotonin [9-11]. Further future prospective studies are needed to investigate the potential effects of preischemic stroke psychotropic medications targeting more than one neurotransmitter (such as 
TABLE 3: Outcome events within 365 days from the initial ischemic stroke.

\begin{tabular}{|c|c|c|c|c|}
\hline \multicolumn{5}{|c|}{ Outcome events within 365 days } \\
\hline & Control $(n=168)$ & Prepsychotropic use $(n=58)$ & Postpsychotropic use $(n=20)$ & $P$ value \\
\hline NIHSS & $0(0-2)$ & $1(0-3)$ & $1.5(0-6)$ & 0.035 \\
\hline Delta NIHSS* & $1(0-4)$ & $3(0-6)$ & $5(0-9)$ & 0.086 \\
\hline Good clinical recovery & $140(82.8 \%)$ & $48(82.8 \%)$ & $16(80.0 \%)$ & 0.891 \\
\hline mRS & $1(0-2)$ & $2(0-3)$ & $2(1-3)$ & 0.013 \\
\hline MACE & $18(10.7 \%)$ & $6(10.3 \%)$ & $1(5.0 \%)$ & 0.831 \\
\hline Myocardial infarction & $2(1.2 \%)$ & $1(1.7 \%)$ & $0(0.0 \%)$ & 1.000 \\
\hline TIA or stroke & $15(8.9 \%)$ & $5(8.6 \%)$ & $1(5.0 \%)$ & 1.000 \\
\hline Cardiovascular death & $1(0.6 \%)$ & $0(0.0 \%)$ & $0(0.0 \%)$ & 1.000 \\
\hline
\end{tabular}

Data are median (IQ range) and $n$ (\%); MACE: major adverse cardiovascular event. $*$ Higher numbers indicate greater improvement from discharge.

SNRI targeting both serotonin and norepinephrine reuptake, for example) on the effects of long-term poststroke recovery.

The strengths of the study were independent reassessment of the clinical diagnoses by a trained neurologist, additional adjudication of uncertain cases by a double boardcertified vascular neurologist and psychiatrist, and the case resolution by a general agreement by the group. An expectation bias was attempted to be minimized by classifying the study groups, blind to any follow-up data. Our study limitations are related to the retrospective study design, its relatively small sample size, and inclusion of the study population from a single tertiary care center, which may have attributed to bias. Another limitation includes lack of specific duration of the psychotropic medication use history in PPMU and PoMU. Our use of mRS as the outcome measures of stroke recovery can be potentially viewed as a limitation. However, the mRS is well known to be a reliable and valid measure of functional outcome [30-32] and has been used in a large, pragmatic clinical trial [13]. Other limitations include lack of patient reported outcome utilizations, including depression screen (e.g., PHQ-2), and lack of a stroke-specific, performance-based impairment index, such as the FuglMeyer assessment. However, these were impractical due to the retrospective study design.

\section{Conclusion}

PPMU in ischemic stroke is common; it can be beneficial in ischemic stroke in the long-term clinical outcome and is not associated with increased risks of MACE. Further studies are warranted to explore the effects of psychotropic medications in ischemic stroke.

\section{Data Availability}

The data used to support the findings of this study are available from the corresponding author upon request to investigators who have received ethical clearance from their host institution review board.

\section{Disclosure}

The content is solely the responsibility of the authors and does not necessarily represent the official views of the
National Institutes of Health. Dr. Silver receives compensation as a surveyor for the Joint Commission, for review of medicolegal malpractice cases, and for adjudication of stroke outcomes in the Women's Health Initiative. We acknowledge that the abstract of this original research paper was presented at the 2019 International Stroke Conference as a poster presentation [33].

\section{Conflicts of Interest}

The authors declare that there is no conflict of interest regarding the publication of this article.

\section{Authors' Contributions}

Adalia H. Jun-O'Connell contributed in the study concept and design, data acquisition, interpretation of the data, drafting of the article, and critical revision of the manuscript for important intellectual content. Dilip K. Jayaraman contributed in the study concept and design, data acquisition, interpretation of the data, and critical revision of the manuscript for important intellectual content. Nils Henninger contributed in the study concept and design, statistical analysis and interpretation of the data, drafting of the article, and critical revision of the manuscript for important intellectual content. Brian Silver contributed in the interpretation of data and critical revision of the manuscript for important intellectual content. Majaz Moonis contributed in the interpretation of the data and critical revision of the manuscript for important intellectual content. Anthony J Rothschild contributed in the study concept and design, interpretation of the data, and critical revision of the manuscript for important intellectual content.

\section{Acknowledgments}

Dr. Henninger is supported by K08NS091499 from the National Institute of Neurological Disorders and Stroke of the National Institutes of Health and R44NS076272 from the Eunice Kennedy Shriver National Institute of Child Health and Human Development of the National Institutes of Health. Anthony J Rothschild was funded by grant/research/clinical trial support from NIMH (psychotic depression (sertraline, olanzapine)), Janssen (depression (esketamine)), and Allergan 
(depression (rapastinel)) and consultant/advisory boards from GlaxoSmithKline (depression, suicide (paroxetine)), Alkermes (depression (ALK-5461)), Sage Therapeutics (depression (sage-217)), and Janssen (depression (esketamine)).

\section{References}

[1] W. Johnson, O. Onuma, M. Owolabi, and S. Sachdev, "Stroke: a global response is needed," Bulletin of the World Health Organization, vol. 94, no. 9, pp. 634-634A, 2016.

[2] E. J. Benjamin, P. Muntner, A. Alonso et al., "Heart disease and stroke Statistics-2019 update: a report from the American Heart Association," Circulation, vol. 139, no. 10, pp. e56e528, 2019.

[3] S. C. Cramer, "Drugs to enhance motor recovery after stroke," Stroke, vol. 46, no. 10, pp. 2998-3005, 2015.

[4] L. B. Goldstein, "Effects of amphetamines and small related molecules on recovery after stroke in animals and man," Neuropharmacology, vol. 39, no. 5, pp. 852-859, 2000.

[5] A. Bueno-Nava, S. Montes, P. DelaGarza-Montano, A. AlfaroRodriguez, A. Ortiz, and R. Gonzalez-Pina, "Reversal of noradrenergic depletion and lipid peroxidation in the pons after brain injury correlates with motor function recovery in rats," Neuroscience Letters, vol. 443, no. 1, pp. 32-36, 2008.

[6] L. B. Goldstein, "Neurotransmitters and motor activity: effects on functional recovery after brain injury," NeuroRx, vol. 3, no. 4, pp. 451-457, 2006.

[7] D. J. Gladstone and S. E. Black, "Enhancing recovery after stroke with noradrenergic pharmacotherapy: a new frontier?," The Canadian Journal of Neurological Sciences, vol. 27, no. 2, pp. 97-105, 2000.

[8] B. B. Bhakta, S. Hartley, I. Holloway et al., "The DARS (Dopamine Augmented Rehabilitation in Stroke) trial: protocol for a randomised controlled trial of co-careldopa treatment in addition to routine NHS occupational and physical therapy after stroke," Trials, vol. 15, no. 1, p. 316, 2014.

[9] L. E. Ramos-Languren, R. Gonzalez-Pina, S. Montes et al., "Sensorimotor recovery from cortical injury is accompanied by changes on norepinephrine and serotonin levels in the dentate gyrus and pons," Behavioural Brain Research, vol. 297, pp. 297-306, 2016.

[10] V. Windle and D. Corbett, "Fluoxetine and recovery of motor function after focal ischemia in rats," Brain Research, vol. 1044, no. 1, pp. 25-32, 2005.

[11] F. Chollet, J. Tardy, J. F. Albucher et al., "Fluoxetine for motor recovery after acute ischaemic stroke (FLAME): a randomised placebo-controlled trial," Lancet Neurology, vol. 10, no. 2, pp. 123-130, 2011.

[12] S. K. McCann, C. Irvine, G. E. Mead et al., "Efficacy of antidepressants in animal models of ischemic stroke: a systematic review and meta-analysis," Stroke, vol. 45, no. 10, pp. 30553063, 2014.

[13] Focus Trial Collaboration, "Effects of fluoxetine on functional outcomes after acute stroke (Focus): a pragmatic, doubleblind, randomised, controlled trial," The Lancet, vol. 393, no. 10168, pp. 265-274, 2019.

[14] K. L. Kraglund, J. K. Mortensen, E. L. Grove, S. P. Johnsen, and G. Andersen, "TALOS: a multicenter, randomized, doubleblind, placebo-controlled trial to test the effects of citalopram in patients with acute stroke," International Journal of Stroke, vol. 10, no. 6, pp. 985-987, 2015.
[15] E. M. O. T. I. O. N. Investigators, "Efficacy of early administration of escitalopram on depressive and emotional symptoms and neurological dysfunction after stroke: a multicentre, double-blind, randomised, placebo-controlled study," Lancet Psychiatry, vol. 4, no. 1, pp. 33-41, 2017.

[16] J. K. Mortensen, H. Larsson, S. P. Johnsen, and G. Andersen, "Impact of prestroke selective serotonin reuptake inhibitor treatment on stroke severity and mortality," Stroke, vol. 45, no. 7, pp. 2121-2123, 2014, Epub 2014 Jun 3.

[17] http://www.strobe-statement.org.

[18] R. L. Sacco, S. E. Kasner, J. P. Broderick et al., "An updated definition of stroke for the 21st century: a statement for healthcare professionals from the American Heart Association/American Stroke Association," Stroke, vol. 44, no. 7, pp. 2064-2089, 2013.

[19] B. Sadock, V. Sadock, and N. Sussman, Kaplan \& Sadock's Pocket Handbook of Psychaitric Drug Treatment, Lippincott Williams \& Wilkins, Philadelphia, 4th edition edition, 2006.

[20] T. Siepmann, J. Kepplinger, C. Zerna et al., "The effects of pretreatment versus de novo treatment with selective serotonin reuptake inhibitors on short-term outcome after acute ischemic stroke," Journal of Stroke and Cerebrovascular Diseases, vol. 24, no. 8, pp. 1886-1892, 2015.

[21] N. Henninger, E. Lin, S. P. Baker, A. K. Wakhloo, D. Takhtani, and M. Moonis, "Leukoaraiosis predicts poor 90-day outcome after acute large cerebral artery occlusion," Cerebrovascular Diseases, vol. 33, no. 6, pp. 525-531, 2012.

[22] A. H. Jun-O'connell, N. Henninger, M. Moonis, B. Silver, C. Ionete, and R. P. Goddeau, "Recrudescence of old stroke deficits among transient neurological attacks," The Neurohospitalist, vol. 9, no. 4, pp. 183-189, 2019.

[23] S. A. Ayis, A. G. Rudd, L. Ayerbe, and C. D. A. Wolfe, "Sex differences in trajectories of depression symptoms and associations with 10-year mortality in patients with stroke: the South London Stroke Register," European Journal of Neurology, vol. 26, no. 6, pp. 872-879, 2018.

[24] C. Carcel, et al.X. Wang, E. C. Sandset et al., "Sex differences in treatment and outcome after stroke," Neurology, vol. 93, no. 24, pp. e2170-e2180, 2019.

[25] B. Poynter, M. Shuman, N. Diaz-Granados, M. Kapral, S. L. Grace, and D. E. Stewart, "Sex differences in the prevalence of post-stroke depression: a systematic review," Psychosomatics, vol. 50, no. 6, pp. 563-569, 2009.

[26] R. C. Malenka and M. F. Bear, "LTP and LTD: an embarrassment of riches," Neuron, vol. 44, no. 1, pp. 5-21, 2004.

[27] R. C. Malenka and R. A. Nicoll, "Long-term potentiation-a decade of progress?," Science, vol. 285, no. 5435, pp. 1870 1874, 1999.

[28] D. M. Feeney and R. L. Sutton, "Pharmacotherapy for recovery of function after brain injury," Critical Reviews in Neurobiology, vol. 3, no. 2, pp. 135-197, 1987.

[29] D. M. Feeney, M. P. Weisend, and A. E. Kline, "Noradrenergic pharmacotherapy, intracerebral infusion and adrenal transplantation promote functional recovery after cortical damage," Journal of Neural Transplantation and Plasticity, vol. 4, no. 3, pp. 199-213, 1993.

[30] A. Bruno, N. Shah, C. Lin et al., "Improving modified Rankin Scale assessment with a simplified questionnaire," Stroke, vol. 41, no. 5, pp. 1048-1050, 2010.

[31] A. Bruno, A. E. Akinwuntan, C. Lin et al., "Simplified modified Rankin Scale questionnaire: reproducibility over the telephone 
and validation with quality of life," Stroke, vol. 42, no. 8, pp. 2276-2279, 2011.

[32] M. Dennis, G. Mead, F. Doubal, and C. Graham, "Determining the modified Rankin score after stroke by postal and telephone questionnaires," Stroke, vol. 43, no. 3, pp. 851-853, 2012.

[33] D. Jayaraman, N. Henninger, B. Silver, M. Moonis, A. Rothschild, and A. H. Jun-Oconnell, "Abstract TP150 : effects of pre-existing psychotropic medication use on a cohort of patients with ischemic stroke outcome," Stroke, vol. 51, Supplement 1, 2020. 